\title{
Estudio de la interacción de luz con sustancias con alto contenido de sacarosa y fructuosa
}

\section{Study of the light interaction with substances high in fructose and sucrose}

GÓMEZ-VIEYRA, Armando ${ }^{1} \dagger^{*}$, COSME-CISNEROS, Ismael Gabriel ${ }^{2}$, MIRANDA-TELLO, José Raúl $^{1}$ y HERNÁNDEZ-MOTA, Sonia ${ }^{1}$

${ }^{I}$ Universidad Autónoma Metropolitana-Unidad Azcapotzalco, División de Ciencias Básicas e Ingeniería

${ }^{2}$ Instituto Politécnico Nacional, Escuela Superior de Ingeniería Mecánica y Eléctrica-Zacatenco

ID 1 ${ }^{\mathrm{er}}$ Autor: Armando, Gómez-Vieyra / ORC ID: 0000-0003-0290-1518, CVU CONACYT ID: 170266

ID 1 ${ }^{\text {er }}$ Coautor: Ismael Gabriel, Cosme-Cisneros / ORC ID: 0000-0003-4036-9753, CVU CONACYT ID: 416331

ID $2^{\text {do }}$ Coautor: José Raúl, Miranda-Tello / ORC ID: 0000-0002-5888-5732, CVU CONACYT ID: 101411

ID $3^{\text {er }}$ Coautor: Sonia, Hernández-Mota / ORC ID: 0000-0001-9096-0467

DOI: $10.35429 / J T I P .2019 .8 .3 .1 .7$

Recibido 03 de Julio, 2019; Aceptado 09 Septiembre, 2019

\section{Resumen}

En el proceso de enseñanza-aprendizaje en los niveles medio superior y superior, es complicado motivar a los estudiantes sobre la importancia de conocer y aplicar los conceptos básicos en los procesos industriales. De aquí la importancia de aplicar el concepto de polarización de la luz y la espectroscopía para visualizar las diferencias entre sustancias a base de sacarosa o de fructuosa. Ambas técnicas se han aplicado en diversos procesos industriales, su costo regularmente es alto y requieren una capacitación amplia. En este trabajo se presentan los resultados de la implementación de un polariscopio lineal de bajo costo para el análisis de miel de abeja natural y jarabes a base de sacarosa. El arreglo consta de un láser semiconductor, un fotodiodo, dos polarizadores, un motor a pasos y algunos elementos mecánicos, todos controlados por la computadora mediante una interfaz gráfica. Para validar los resultados se emplea un arreglo óptico basado en un espectroscopio comercial de bajo costo. Al implementar este arreglo experimental, se involucran los conceptos de polarización, actividad óptica, física atómica-molecular, instrumentación electrónica y principios de diseño y construcción de equipo.

Miel, Polarización, Actividad óptica

\begin{abstract}
In the teaching-learning process at the upper and upper secondary levels, it is difficult to motivate students about the importance of knowing and applying basic concepts in industrial processes. Therefore, it is proposed how to apply the concept of light polarization and spectroscopy to visualize the differences between substances based on sucrose or fructose. Both techniques have been applied in various industrial processes; their cost is regularly high and require extensive training. In this paper, we present the results of the implementation of a low cost linear polariscope for the analysis of natural bee honey and syrups based on sucrose. The arrangement consists of a semiconductor laser, a photodiode, two polarizers, a stepper motor and some mechanical elements, all controlled by the computer through a graphical interface. To validate the results, an optical array based on a lowcost commercial spectroscope is used. By implementing this experimental arrangement, the concepts of polarization, optical activity, atomic-molecular physics, electronic instrumentation and principles of equipment design and construction are involved.
\end{abstract}

Honey, Polarization, Optical activity

Citación: GÓMEZ-VIEYRA, Armando, COSME-CISNEROS, Ismael Gabriel, MIRANDA-TELLO, José Raúl y HERNÁNDEZ-MOTA, Sonia. Estudio de la interacción de luz con sustancias con alto contenido de sacarosa y fructuosa. Revista de Tecnologías en Procesos Industriales. 2019 3-8: 1-7

\footnotetext{
* Correspondencia del Autor (Correo electrónico: agvte@azc.uam.mx)

$\dagger$ Investigador contribuyendo como primer autor.
} 


\section{Introducción}

La miel se entiende como una sustancia dulce de origen natural producida por abejas. Estas se encargan de transportar, combinar y transformar con sustancia propias el néctar de las flores o de secreciones de algunas plantas que almacenan en el panal hasta que esta madure y esté apta para su consumo.

Los azúcares que no son procesados por la abeja no son mieles, por lo que resulta suficiente con el solo considerar la de miel de abeja. Por otro lado, su origen botánico confiere las características aromáticas, de color, consistencia y sabor; esta amplia variedad de mieles hace que la identificación de una miel sea una labor difícil $[1,2]$.

Las exigencias en la calidad, así como las pruebas necesarias para su autentificación están descritas en las normas mexicanas NOM-F-0361981 y NOM-051-SCFI-1994.

En estas se realizan diversos estudios físico-químicos para su total validación como una miel auténtica, cabe mencionar que las normas que rigen la calidad de la miel han sido tomadas de documentos ya existentes de normas de diferentes países.

Desde el punto de vista químico, la miel es una solución sobresaturada de azúcares, que tienden a precipitarse; es decir, a formar cristales sólidos según las proporciones de glucosa, fructosa y agua [3]. Debido a esto, se ha demostrado que, empleando luz polarizada, es posible realizar una caracterización de esta [4].

La polarización de la luz fue descubierta por E. L. Malus [5,6]. Esta teoría describe y analiza las propiedades de la luz al interaccionar con algunos materiales.

La polarización se puede describir por medio de una orientación específica del campo eléctrico en un periodo de oscilación, al interaccionar este haz de luz polarizada con un material su orientación puede variar o no $[7,8]$.

El estudio de las propiedades de la polarización óptica de los materiales es de suma importancia para la identificación de algunos productos comerciales como: aceite de hierbabuena, aceite de comino, sustancias basadas en sacarosa y fructuosa, entre otros [9].
Estas sustancias presentan actividad óptica natural propia, lo cual quiere decir que al incidir un haz de luz polarizado linealmente (con cualquier orientación) presenta una rotación óptica, es decir, el ángulo de polarización de la luz gira, ya sea a la derecha (dextrógira) o a la izquierda (levógira). Esta propiedad se mesura al implementar un polarímetro lineal, el cual puede ser automatizado, con el fin de distinguir ciertas propiedades de la luz transmitida.

En la instrumentación química es común medir la rotación específica, definida como:

$[\alpha]=\frac{\alpha}{c l}$

Donde $\alpha$ es la rotación en grados, $c$ es la concentración en $\mathrm{g} / \mathrm{ml}$ y $l$ la longitud de la cavidad en m. En el campo de la enseñanza de la física, hemos considerado que es más pedagógico para la formación y asimilación del conocimiento, el observar el desfasamiento entre los datos experimentales en la medición de la rotación óptica ya que permite el mejor análisis de este fenómeno.

Esto permite observar diferencias entre la rotación dextrógira y levógira, y mediante estas mediciones es posible discernir entre elementos con diferente actividad óptica especifica. Por ejemplo, esta técnica tiene el potencial, al ser correlacionada con otras, de:

- Diferenciar la región de origen de mieles $100 \%$ naturales.

- Discernir entre una miel natural y un jarabe con sacarosa.

- Identificar cuando esté presente alguna alteración de un lote de productos.

- Clasificar los efectos por absorción.

Por otro lado, es posible diferenciar los diversos compuestos de una sustancia al observar sus espectros de absorción, reflexión y transmisión. A la implementación de estas técnicas comúnmente se les denominan espectrofotometría.

En específico se han realizado trabajos de investigación en la región del infrarojo para mieles naturales y jarabes sacarosos, que han demostrado ser técnicas muy efectivas para vericar la naturaleza de estas sustancias. 
Con la combinación de la técnica de polarimetría y la de espectrofotometría por tansmisión en el visible e IR cercano, se espera tener la capacidad de discernir entre una sustancia sacarosa, fructuosa o alterada.

\section{Arreglo experimental}

\section{a) Medición del ángulo de la polarización lineal}

Un esquema a bloques del arreglo experimental simplificado para realizar las mediciones de la polarización se muestra en la Figura 1. Las partes más importantes son: un Diodo Láser, el cual tiene una longitud de onda de 663nm, alimentado por una fuente de corriente tipo Howland a $15.5 \mathrm{~mA}$ y una resistencia de carga de $220 \Omega$.

Dos polarizadores Newport 10P009AR.14 con un rango de operación de 630-700nm y montados en dos bases rotatorias manuales Newport RSPT-1T, que además fueron modificadas para añadir el control del movimiento automatizado. El motor a pasos empleado es un NEMA 17; además, se empleó un controlador comercial A4988 y es controlado por una tarjeta Arduino UNO.

Este sistema de desarrollo cumple la función de muestrear la etapa de detección, de transmitir la información a la computadora personal y de controlar el sistema experimental. El segundo polarizador, que tiene la función de analizador, permanece fijo, mientras el primer polarizador lineal es desplazado para cambiar el ángulo de polarización incidente de la muestra. Para lograr esto, se diseñaron y fabricaron las monturas y acoples necesarios por medio de impresión en 3D.

La etapa de detección consiste en un fotodiodo TEPT5700, cuyo pico de sensibilidad está en $550 \mathrm{~nm}$ y en el intervalo de 600 a $700 \mathrm{~nm}$ presenta un mínimo de sensibilidad relativa de $75 \%$, acoplado a un amplificador de transimpedancia, Imax de 26mA y Vmax de salida $4.5 \mathrm{~V}$, que es muestreado digitalmente por la tarjeta Arduino UNO.

La computadora guarda la información en un archivo del formato ".txt", que posteriormente es procesado para el análisis de los resultados. Las sustancias puras analizadas se muestran en la Figura 2.

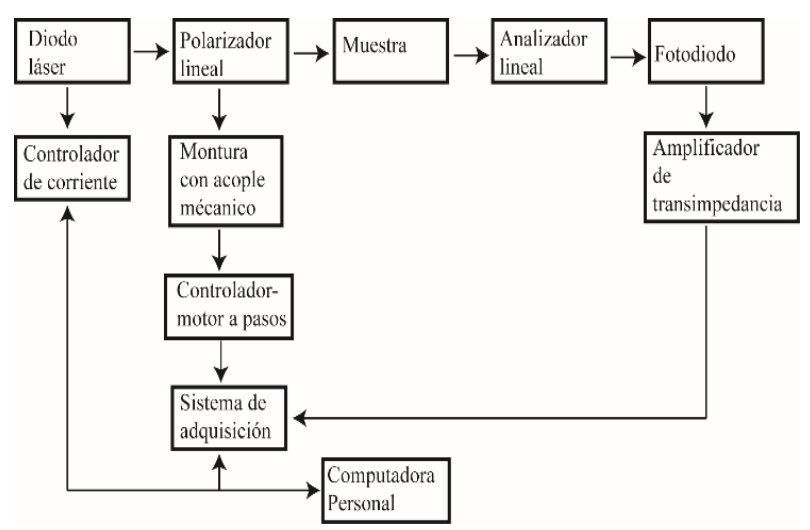

(a)

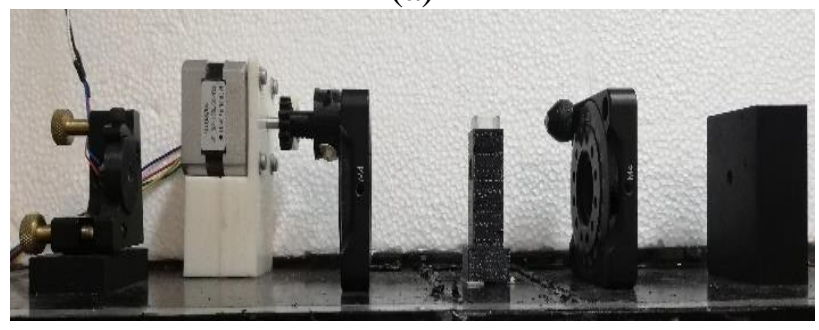

(b)

Figura 1 a) Diagrama a bloques del polarímetro lineal para analizar sustancias con fructuosa y sacarosa. b) Arreglo experimental

Fuente: Elaboración Propia

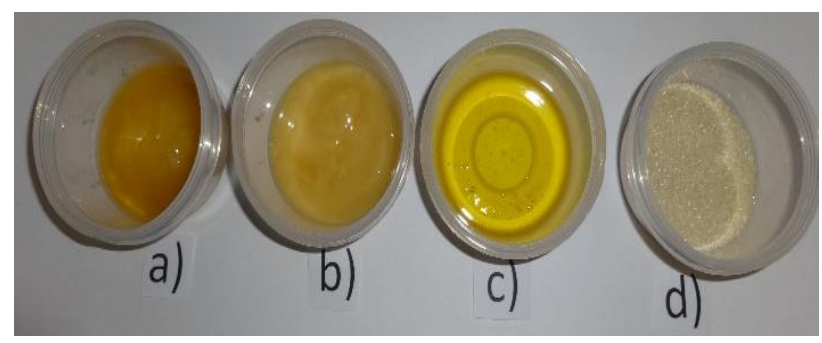

Figura 2 a) Miel de Zacatecas. b) Miel de Oaxaca. c) Jarabe de azúcar tipo miel. d) Azúcar morena comercial Fuente: Elaboración Propia

\section{b) Programa de control y detección}

El cerebro del sistema experimental es un programa en lenguaje gráfico que realiza la medición del voltaje presente en el fotodiodo, controla el tamaño de paso del motor y el almacenamiento de archivos de datos en formato txt.

El diagrama de flujo se muestra en la Figura 3, donde primero se verifica el correcto funcionamiento del láser, se leen los parámetros de entrada para el control (tamaño de paso y ángulo de referencia) y realización de la medición (tamaño de paso, definición de referencia, ruta de la dirección del archivo donde se guardarán los datos y nombre del archivo de almacenamiento). 
A continuación, se mide la diferencia de potencial en el detector, empleando el convertidor analógico digital del sistema de desarrollo electrónico y se realiza la conversión a corriente detectada por el fotodiodo, para ser almacenándos en el archivo ".txt" el dato de la medición y el ángulo donde se realizó. Se procede a alimentar el motor a pasos para que realice el desplazamiento preestablecido, se actualiza el contador del ángulo actual de la montura y se compara con el ángulo de referencia; si son iguales, se termina el proceso de medición.

\section{c) Estudio espectrofotométrico}

Para poder estudiar el espectro de transmisión de la luz, se ensambló un sistema experimental cuya configuración a bloques corresponde a la Figura 4, el cual consta de una lámpara comercial modelo MSR700 como fuente de luz, la muestra que es contenida por una celda de espectroscopía, un espectrómetro 3B Scientific U1710 conectado a una computadora personal por un puerto USB. Con el software del fabricante se realizaron los muestreos correspondientes para poder analizar los datos.

\section{Análisis de resultados}

En una corrida completa del giro de $360^{\circ}$ del analizador, el sistema entrega datos entre $0 \mathrm{~V}$ y $5 \mathrm{~V}$, que es el rango del subsistema de detección, estos son graficados en la Figura 5. Debido a que es muy complicado analizar el conjunto completo de datos, en este trabajo se presentan datos entre $80^{\circ}$ y $190^{\circ}$.

La Figura 5 presenta la respuesta al sistema al tener como muestra agua potable embotellada que se empleo en las soluciones analizadas. Todas las muestras se disolvieron y analizaron con el mismo tipo de agua.

La Figura 6 muestra los resultados obtenidos para el azúcar y jarabe a base de azúcar tipo miel, disueltos con agua en razón 1 a 2. En esta figura se observa claramente el desplazamiento a la izquierda respecto al agua, los datos están normalizados debido a que hay absorción de luz debido a la sustancia disuelta.

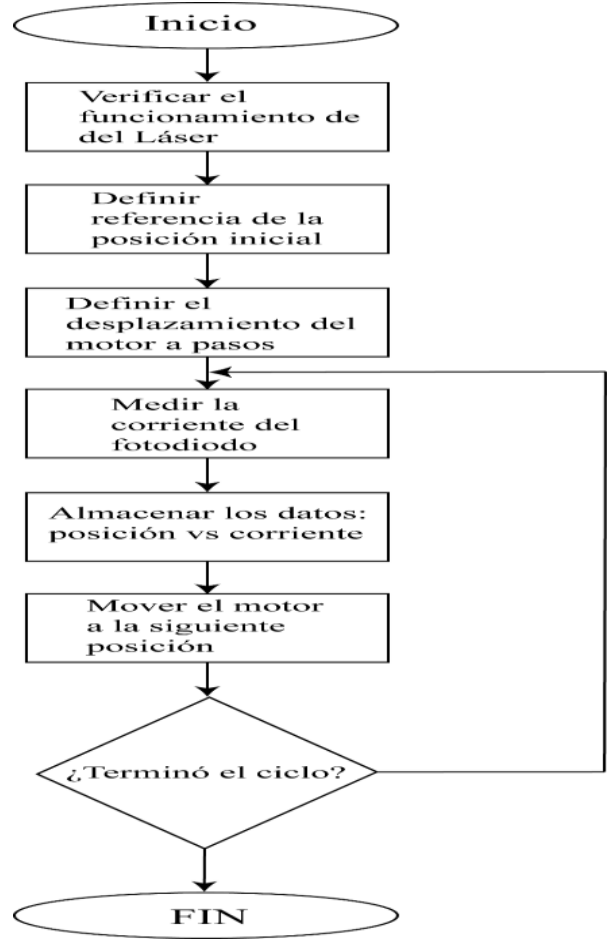

Figura 3 Diagrama de flujo del programa de control del arreglo polarimétrico experimental

Fuente: Elaboración Propia

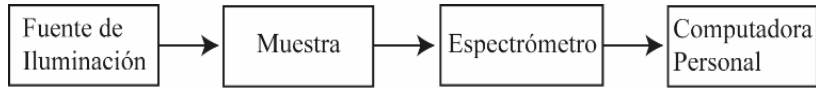

(a)

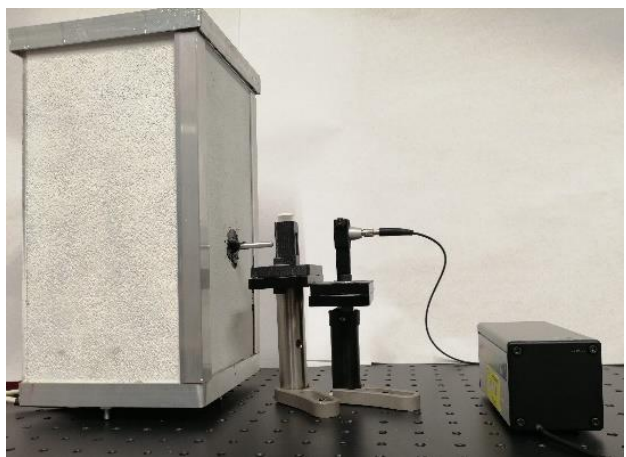

(b)

Figura 4 a) Diagrama del arreglo experimental para el análisis del espectro de absorción de sustancias con fructuosa y sacarosa. b) Foto del arreglo experimental Fuente: Elaboración Propia

La Figura 7 muestra los resultados de mieles puras obtenidas directamente de productores locales de la zona de Zacatecas y de la zona de Oaxaca, ambos están disueltos en agua en relación de 1 a 2 , en esta gráfica se observa claramente un corrimiento a la derecha.

La Figura 8 muestra los resultados obtenidos de una mezcla miel-azúcar al 50\%, disuelta en agua a una razón 1 a 2, en esta gráfica se observa claramente un corrimiento a la izquierda de la mezcla respecto la miel base de cada mezcla. 
Con estos resultados se verifica que el arreglo experimental propuesto permite mesurar los efectos producidos por la actividad óptica de la sustancia sacarosa y fructuosa. También permite observar los efectos de mezclar ambos. Sin embargo, la prueba no es definitiva para verificar la autenticidad de miel comercial, lo cual se puede complementar con el estudio de la espectroscopía de la sustancia.

Además, nos permite proponer un prototipo que puede emplearse tanto en el ámbito comercial como en el ámbito académico. Es importante considerar que la celda de vidrio pírex empleada tenía una geometría cuadra de $12 \mathrm{~mm} \times 12 \mathrm{~mm}$. Al considerar la Ec. 1, es conveniente considerar cambiar la geometría de la celda, debido a que entre más larga sea la trayectoria recorrida por la luz polarizada dentro de la muestra, mayor será el efecto final debido a la actividad óptica de la muestra.

Además, se realizó el estudio de transmisión en la región del espectro visible, Figuras 9, 10 y 11, donde se observa que presentan respuestas similares. Las diferencias más notorias se encuentran en la región del rojo a 690nm. Esto nos indica que hay que profundizar en las regiones del infrarrojo. Pero también nos indica que es posible identificar la adulteración o la simulación de una miel por medio de un sistema opticoelctrónico para analizar la transmitancia local en esta región del espectro.

Todo este trabajo puede emplearse para diferentes cursos de instrucción universitaria, proyectos e investigaciones que permitirán comprender conceptos de física moderna y óptica, con esto se refuerza la integración de la ingeniería con la ciencia básica.

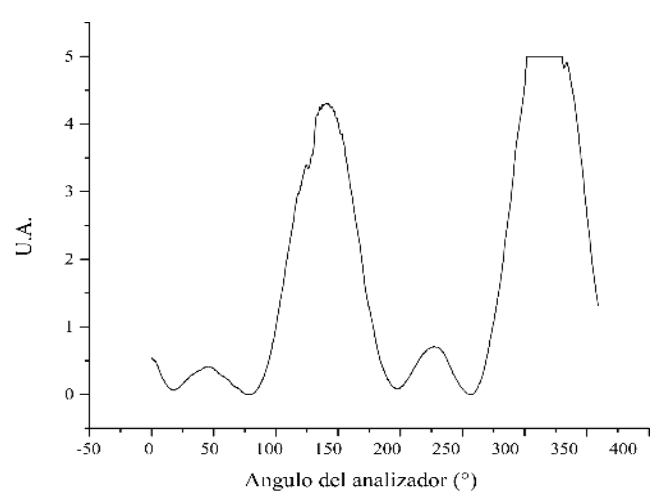

Figura 5 Señal de salida del sistema polarimétrico teniendo como sujeto de muestra agua potable embotellada que se emplea en las soluciones a analizar Fuente: Elaboración Propia

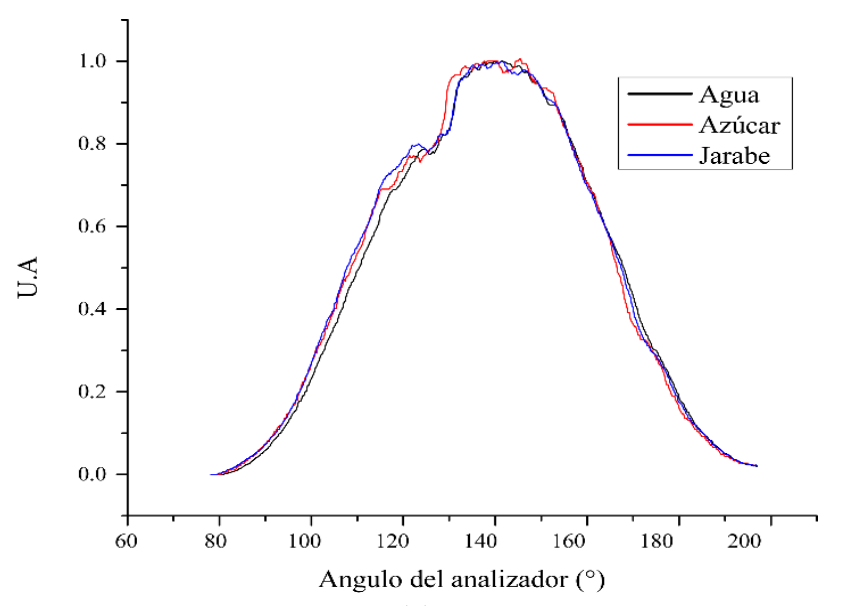

(a)

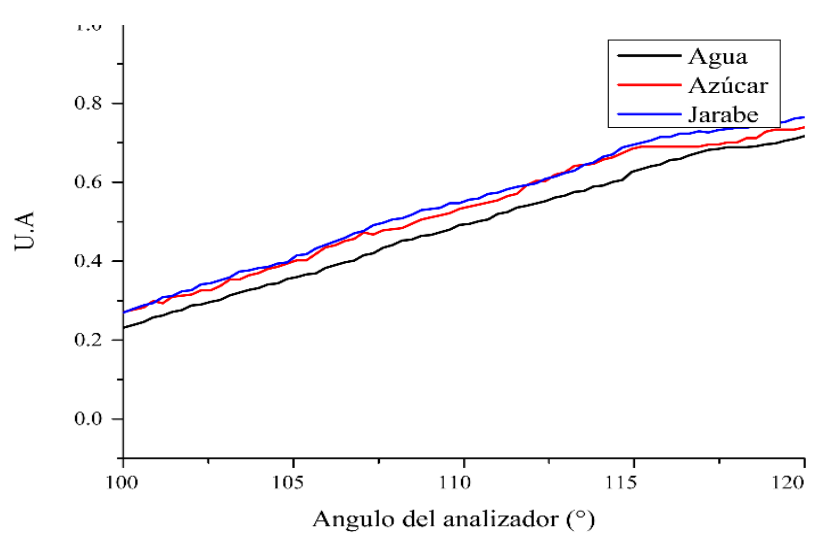

(b)

Figura 6 (a) Evaluación de azúcar y jarabe de azúcar tipo miel disueltos en agua a razón 1:2. (b) Detalle del corrimiento

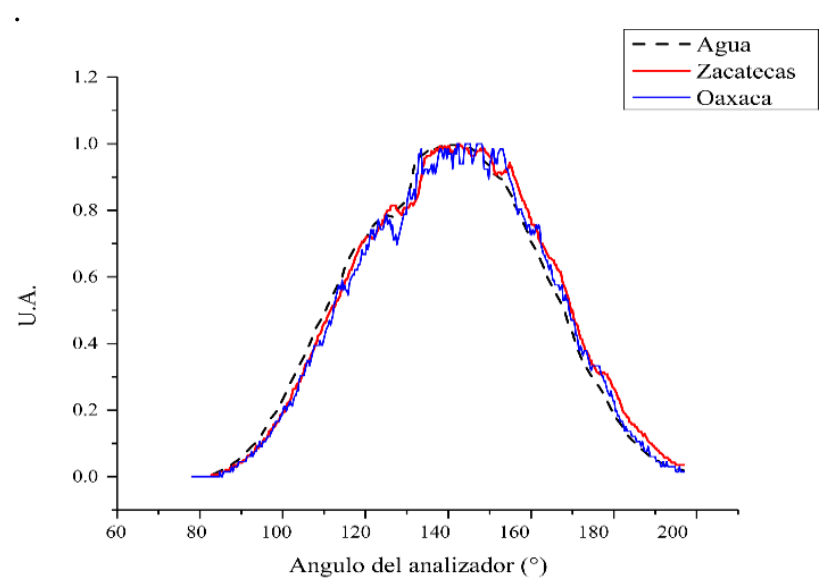

(a)

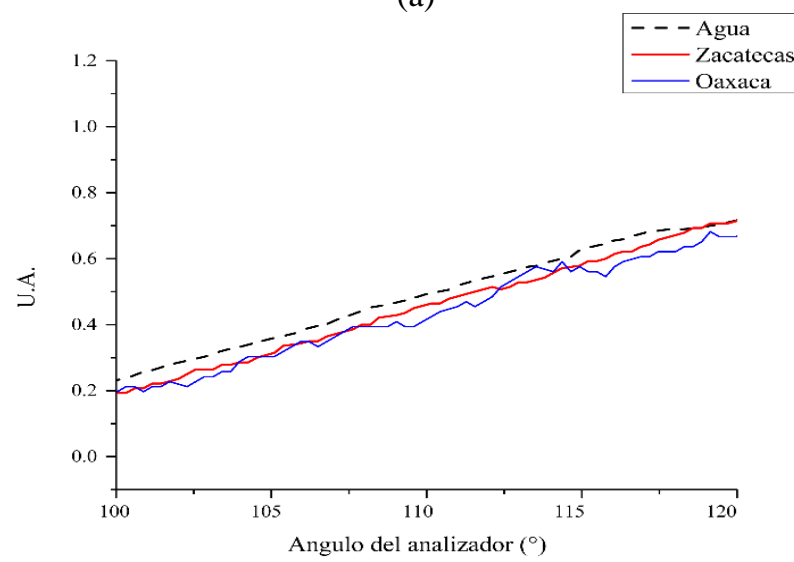

(b)

Figura 7 (a) Evaluación de miel de Zacatecas y Oaxaca disueltos en agua a razón 1:2. (b) Detalle del corrimiento

GÓMEZ-VIEYRA, Armando, COSME-CISNEROS, Ismael Gabriel, MIRANDA-TELLO, José Raúl y HERNÁNDEZ-MOTA, Sonia. Estudio de la interacción de luz con sustancias con alto contenido de sacarosa y fructuosa. Revista de Tecnologías en Procesos Industriales. 2019 


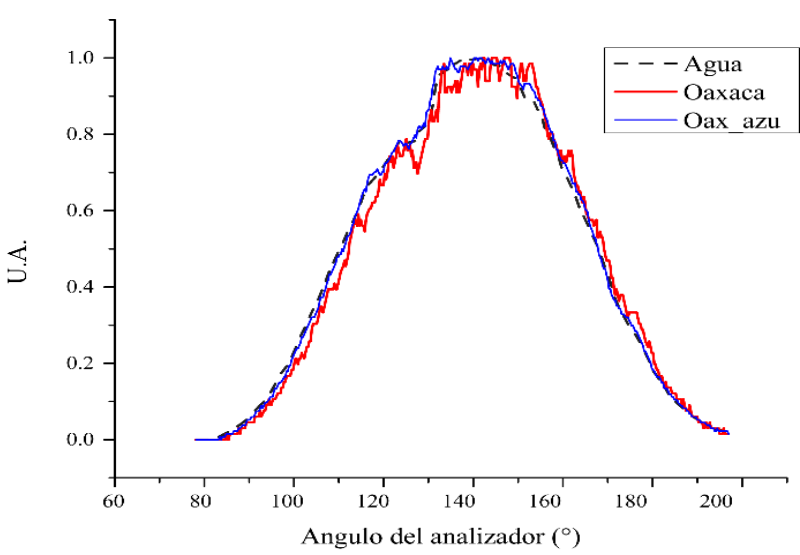

(a)

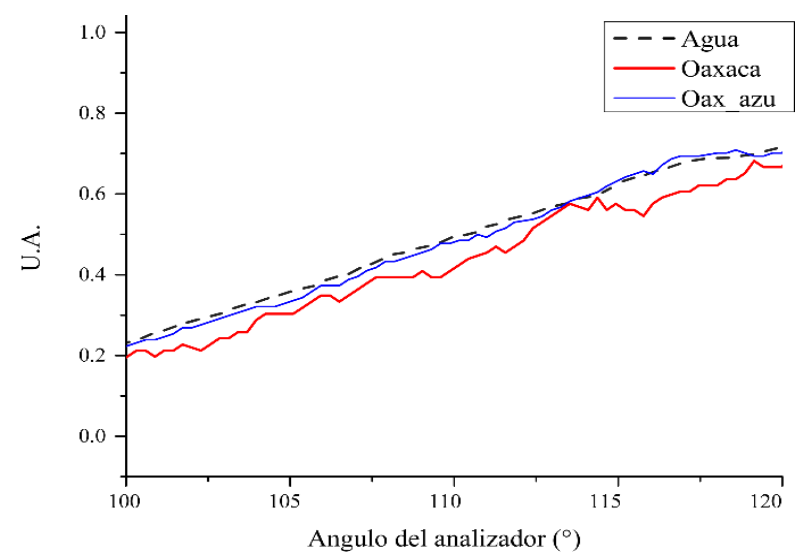

(b)

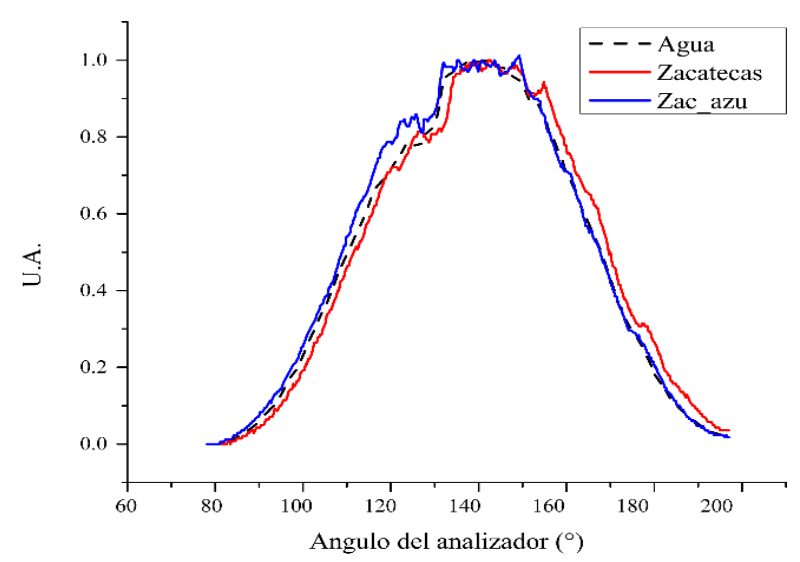

(c)

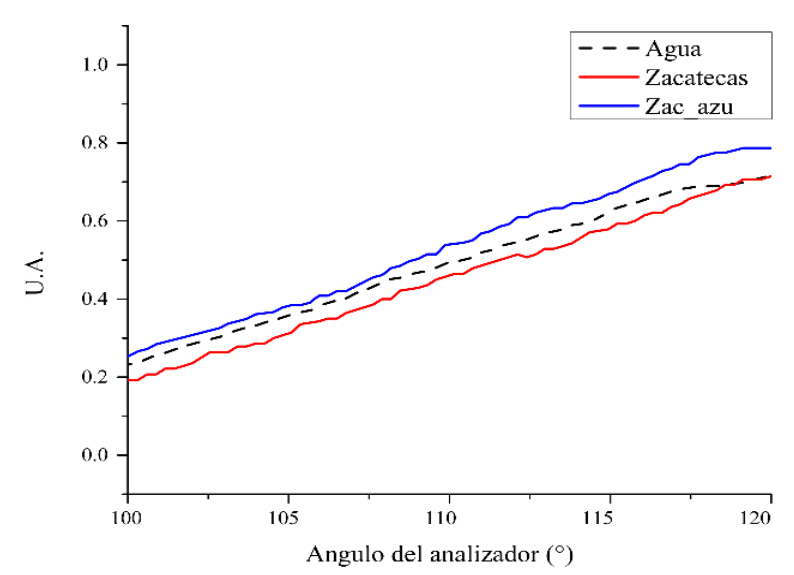

(d)

Figura 8 (a) Evaluación de miel de Oaxaca y mezcla mielazúcar al 50\% disueltos en agua a razón 1:2. (b) Detalle del corrimiento. (c) Evaluación de miel de Zacatecas y mezcla miel-azúcar al 50\% disueltos en agua a razón 1:2. (d) Detalle del corrimiento

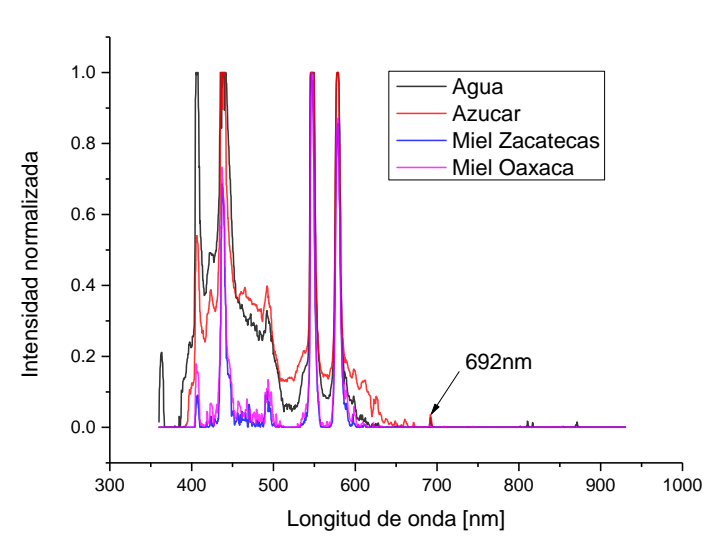

Figura 9 Espectro de transmisión del agua, el azúcar, la miel de Zacatecas y la miel de Oaxaca

Fuente: Elaboración Propia

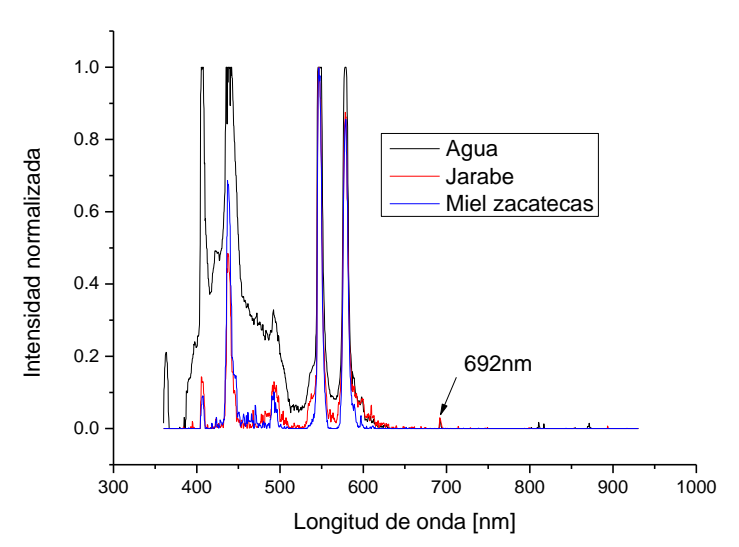

Figura 10 Espectro de transmisión del jarabe de azúcar tipo miel y la miel de Zacatecas Fuente: Elaboración Propia

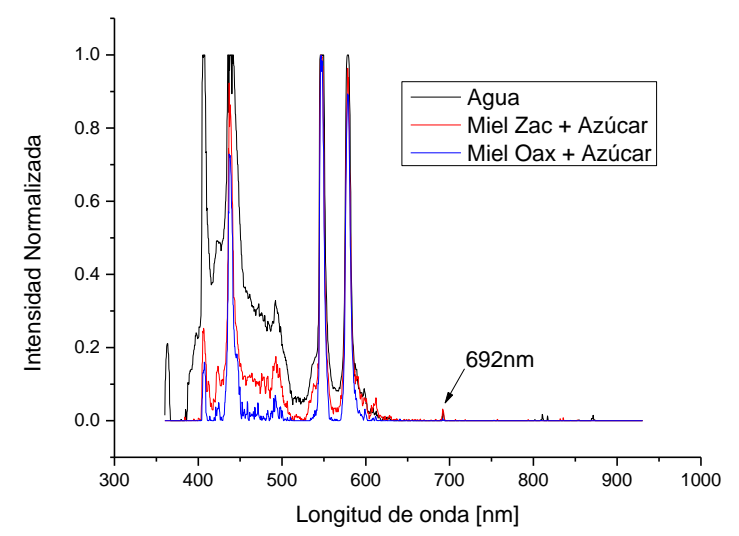

Figura 11 Espectro de transmisión de la mezcla de miel de Zacatecas y la miel de Oaxaca con azúcar Fuente: Elaboración Propia

\section{Conclusiones}

Los resultados obtenidos en artículo muestran que con la investigación básica y aplicada es posible la detección de la adulteración de la miel, seleccionando las técnicas ópticas adecuadas y acotando el espectro de luz para crear prototipos útiles a la sociedad.

GÓMEZ-VIEYRA, Armando, COSME-CISNEROS, Ismael Gabriel, MIRANDA-TELLO, José Raúl y HERNÁNDEZ-MOTA, Sonia. Estudio de la interacción de luz con sustancias con alto contenido de sacarosa y fructuosa. Revista de Tecnologías en Procesos Industriales. 2019 
Además, es importante en trabajos futuros constatar que las técnicas mostradas no son solamente aplicables a sustancias endulzantes, sino pueden ser aplicados en aceites y otros productos. Este trabajo sirve como una guia para ser reproducido o adaptado en el proceso de enseñanza-aprendizaje a nivel superior, por ejemplo en el diseño y construcción de equipos, sistemas de caracterización óptica e instrumentación química.

El empleo de la luz polarizada nos da únicamente un indicativo de si la miel es pura o no. Es notorio que el grado de pureza de la miel observa una actividad óptica distinguible (Figura 7). Del mismo modo una sustancia completamente sacarosa.

Es importante señalar que el análisis espectrofotométrico nos muestra resultados muy interesantes en la frontera del rojo y el infrarrojo, en específico en los 692nm. Sin embargo, es imprescindible estudiarlo en la región del infrarrojo y tal vez del ultra violeta para poder encontrar el ancho de banda adecuado para una mejor discriminación de sustancias con sacarosa, fructuosa o la mezcla de ambas e incluso la adulteración de productos.

\section{Referencias}

[1] Secretaría de Agricultura, P. 2018. La miel en México y el mundo. Recuperado de: https://www.gob.mx/sagarpa/articulos/la-mielen-mexico-y-el-mundo?idiom=es

[2] Laboratorio PROFECO, Estudio de calidad: miel de abeja, Revista del Consumidor, Junio 2011. Recuperado de: http://revistadelconsumidor.gob.mx/?p=19045.

[3] NMX-F-036-981. MIEL DE ABEJA. ESPECIFICACIONES. NORMA MEXICANA. DIRECCIÓN GENERAL DE NORMAS. [Archivo PDF]. Recuperado de: https://www.colpos.mx/bancodenormas/nmexic anas/NMX-F-036-1981.PDF

[4] R. Espinosa-Luna, I. Saucedo-Orozco, C. V. Santiago-Lona, J. M. Franco-Sánchez, A. Magallanes-Luján, "Polarimetric applications to identify bee honey", Proc. of SPIE, vol. 8287 , 82870F, 2011.

[5] E. Hecht, Optics, Addison-Wesley Publishing Co.,2001.
[6] J. M. Strong, Concepts in Classical Optics, Freeman, 1958.

[7] S. Huard, Polarization of light, Wiley, 1997

[8] D. Goldstein, Polarized Light, Marcel Dekker, 2003

[9] W.A. Shurcliff, S.S. Ballard, Luz Polarizada, Reverte, 1968.

WGT. (2002). Waste Gas Technology Energy from Waste. Available at: http://www.wgtuk.com/ukindex.html.

Xiao, R., Jin, B., Zhou, H., Zhong, Z., \& Zhang, M. (2007). Air gasification of polypropylene plastic waste in fluidized bed gasifier. Energy Conversion and Management, 778-786. 